\title{
$L$-shell Filling Rates of Highly Charged Ions in Metals
}

\author{
R. Díez Muiño, N. Stolterfoht, ${ }^{*}$ A. Arnau,${ }^{\dagger}$ A. Salin, ${ }^{\dagger}$ and P. M. Echenique ${ }^{\dagger}$ \\ Departamento de Física de Materiales, Universidad del Pais Vasco/Euskal Herriko Unibertsitatea, Donostia, Euskadi, Spain
} (Received 22 September 1995)

\begin{abstract}
The role of valence electrons in filling inner-shell vacancies of highly charged ions in metals is studied using self-consistent solid state screened potentials. Explicit results for $\mathrm{Ne}$ and $\mathrm{N}$ ions in $\mathrm{Au}$ and $\mathrm{Al}$ targets are presented and discussed in connection to recent experiments. These filling rates are fundamental in understanding the neutralization and relaxation of highly charged ions in solids. [S0031-9007(96)00375-4]
\end{abstract}

PACS numbers: 79.20.Rf, 78.90.+t, 79.90.+b

When a slow multicharged ion approaches a metal surface it may capture electrons into excited states giving rise to the formation of a hollow atom, i.e., an atom whose inner shells remain essentially unoccupied. The relaxation processes that take place as it approaches the surface are complex and still not well understood. Furthermore, the eventual crossing of the surface and penetration into the solid introduce effects that impose many constraints to the theoretical models recently developed to explain the large amount of experimental data [1-9] on emission yields of electrons, photons, and ions, as well as doubly differential electron spectra. Understanding qualitatively and quantitatively the physics under conditions far from stability [10-12] is a major challenge for both experimentalists and theoreticians.

A widely used model to study the relaxation of slow multicharged ions approaching a metal surface is the classical over-the-barrier (COB) model developed by Burgdörfer, Lerner, and Meyer [13]. It predicts the survival of inner-shell vacancies at the instant of crossing the metal surface, and population of $n=4,5$ Rydberg states for $\mathrm{N}, \mathrm{O}$, and $\mathrm{Ne}$ ions. The $\mathrm{COB}$ model explains recent experiments done by Folkerts et al. [14] using oxygen ions under surface channeling conditions on $\mathrm{Au}$ single crystals. On the other hand, up to now very little was known about the relaxation mechanism in the case of insulating targets like LiF. It is not even clear whether the hollow atom is formed as the multicharged ion approaches the solid surface [3].

Recently, much effort has been devoted [15-17] to studies of processes taking place inside the bulk of a metallic target. The formation of a hollow atom in the solid, sometimes called hollow atom of the second generation, with $K$ - and $L$-shell vacancies, involves specific features of the metal electrons that are fast enough to develop a dynamic screening cloud. The shape of this screening cloud is essentially determined by the number of innershell vacancies and not by the initial population of the outer shells. Two mechanisms compete in filling inner shells of first row ions: Auger transitions and resonant capture from target levels (radiative transitions play a role only for heavier ions). When electrons are created inside the solid, the elastic and inelastic scattering of the emitted electrons on their way out of the solid has to be considered to understand the measured spectra.

As far as electronic states are concerned, the main difference between the electron-multicharged ion interaction outside and inside a metal is screening by valence electrons in the solid. An important effect of screening is the reduction of the range of the electron-ion interaction potential; a lower number of bound states is then supported. Another important effect is that charge neutrality in the system is provided by an induced charge which has a bound and a continuum contribution. The latter is larger when inner-shell holes are present and requires a nonperturbative treatment of the interaction. However, the precise knowledge of the potential is a difficult many-body problem [18]. Because of the strong perturbation of the valence electrons by the multicharged ion, particularly in the spatial region within a few atomic units around it, a self-consistent calculation is needed to study this highly nonlinear screening problem.

In this work we calculate, a priori, the $L$-shell filling rates of multicharged ions in metals, which are key ingredients in the theoretical determination of emitted electron spectra. These rates have usually been treated as fitting parameters or taken from atomic Auger data. The process considered here is one in which the role of the outer-shell electrons in the conventional atomic Auger process is played by the conduction band electrons; the collective response of the latter is quite important in this phenomenon [19]. We shall denote the studied Auger processes by LCV (see Fig. 1): The final localized electronic state is an $L$-shell state bound to the ion, the initial continuum electronic state $(\mathrm{C})$ is centered around the ion, and the extended valence electron states (V) are assumed to respond in the process in providing electronhole pair and plasmon excitations. We are interested, in particular, in the evolution of the LCV-Auger rate as a function of the number of $L$-shell electrons $\left(n_{L}\right)$. Both the initial continuum and final bound electronic states are strongly dependent on the screening of the nuclear charge by the $L$-shell electrons, i.e., on $n_{L}$. 


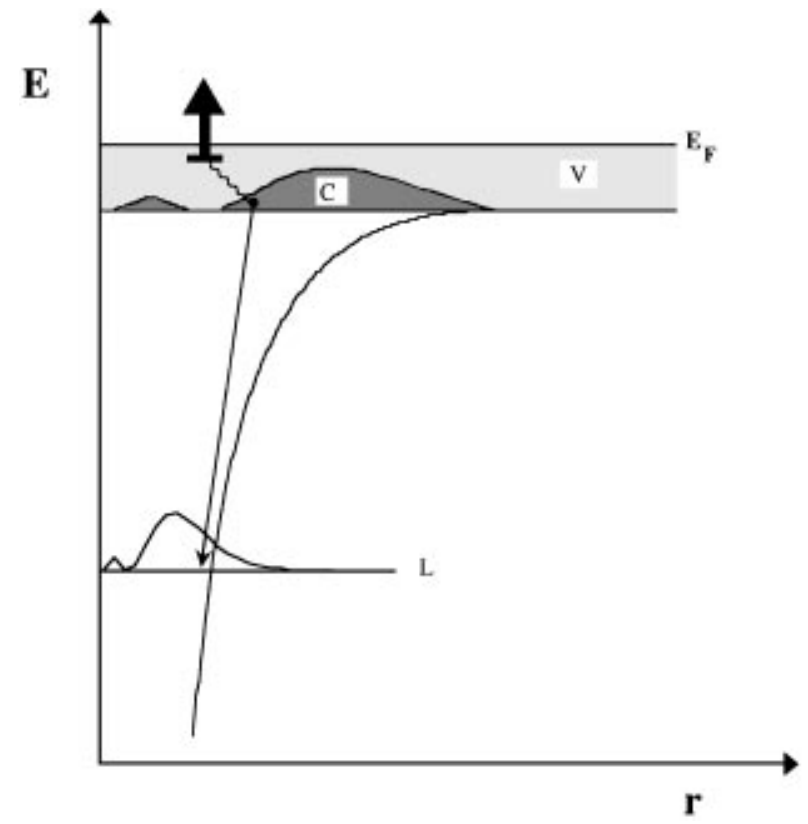

FIG. 1. Schematic picture of the LCV-Auger process: The $\mathrm{C}$ electronic cloud is the induced electronic density in the continuum centered around the ion, while V comes from the background density of the valence electrons. $\mathrm{L}$ is the bound state wave function. In a LCV-Auger process, a conduction band electron jumps to a bound state of the ion, assisted by a third body that may be a plasmon or an electron-hole pair (represented by the thick black arrow).

We have chosen multicharged $\mathrm{N}$ and $\mathrm{Ne}$ ions with one $K$-shell hole in electron gases with density parameters $r_{s}=1.5$ and $r_{s}=2$ to represent $\mathrm{Au}$ and $\mathrm{Al}$ targets [20], respectively. The nonlinear screening problem is treated within the framework of density functional theory [15] as applied to a static impurity in an electron gas [21,22]. Hence, we assume that (i) the ion velocity is much lower than the Fermi velocity of the metal electrons, and (ii) the time scale over which the metal electrons respond to the perturbation $\left(10^{-16} \mathrm{~s}\right)$ is short compared to the typical time scale for the filling by Auger transitions of the inner shells. Our results show that he latter condition is usually fulfilled.

The solution of the Kohn-Sham equations [23] provides us, for a given number of vacancies, with the selfconsistent potential and electron density around the ion, as well as the total energies. The Auger rates per spin state are given (atomic units are used throughout this Letter) by [24]

$$
\begin{aligned}
\Gamma_{\alpha}= & 2 \pi \sum_{k<k_{F}} \int d \omega \int \frac{d^{3} \mathbf{q}}{(2 \pi)^{3}}\left(\frac{4 \pi}{q^{2}}\right)^{2}\left|\left\langle\phi_{\alpha}\left|e^{i \mathbf{q r}}\right| \phi_{k}\right\rangle\right|^{2} \\
& \times S(q, \omega) \delta\left[\omega-\Delta E_{\alpha k}\right],
\end{aligned}
$$

where $k_{F}$ is the Fermi wave number, $\left|\phi_{k}\right\rangle,\left|\phi_{\alpha}\right\rangle$ are the initial continuum and final bound one-electron states, with corresponding energies $E_{k}$ and $E_{\alpha}$, respectively $\left(\Delta E_{\alpha k}=\right.$ $\left.E_{k}-E_{\alpha}\right)$, that are eigenstates of the same Hamiltonian.
The dynamic structure factor $S(q, \omega)$ accounts for the self-consistent response of the valence electrons, represented by a uniform electron gas in our model. Guinea and Flores [25] have shown that the neglect of the orthogonalization hole in the response function is appropriate to calculate Auger rates. Thus, to evaluate $S(q, \omega)$ we have used the wave vector and frequency dependent random phase approximation response function [26]. The function $S(q, \omega)$ measures both the screening and the density of excitations in the perturbed electron gas. In Eq. (1) the one-electron matrix elements take appreciable values only if the overlap between the initial and final states is not too small. In addition, the coupling decreases as the transition energy increases to high enough energies [typically larger than the plasmon energy $\omega_{p}$ (cf. Fig. 3)]. Finally, the summation over occupied states below the Fermi level accounts for the density of states in the continuum and depends on the value of the unperturbed electron density of the metal. These three factors are the key quantities that determine the rates, as our results show.

In Fig. 2 we plot the LCV-Auger rates as a function of the number of $L$-shell electrons in the final state $\left(n_{L}\right)$ for $\mathrm{Ne}$ [Fig. 2(a)] and $\mathrm{N}$ [Fig. 2(b)] ions with one $K$-shell electron for two different background electron densities $\left(r_{s}=1.5\right.$ and $\left.r_{s}=2\right)$. These rates are obtained from the rates per spin state given by Eq. (1), that also depend on $n_{L}$ through the self-consistent potential that determines the bound and continuum one-electron states. The statistics over electron distribution in the final state, for a given $n_{L}$, is performed by assuming that, due to the fast $2 p \rightarrow 2 s$ Coster-Kronig type process, $n_{2 s}=2$ whenever $n_{L} \geq 2$, according to

$$
\begin{gathered}
\Gamma=\left(6-n_{2 p}\right) \Gamma_{2 p}+\left(2-n_{2 s}\right) \Gamma_{2 s}, \\
\text { with } n_{2 s}+n_{2 p}=n_{L} .
\end{gathered}
$$

However, our results are not significantly altered by using a different statistics like the approximation $\Gamma \approx$ $\left(8-n_{L}\right) \Gamma_{2 p}\left(n_{L}\right)$. First changing $\Gamma_{2 s}$ by $\Gamma_{2 p}$ in Eq. (2) modifies $\Gamma$ by less than $15 \%$. Second, the rate per spin state for a given $n_{L}$ does not vary appreciably by changing the subshell distribution.

In the case of Ne hollow atoms the LCV-Auger filling rates are weakly dependent on $n_{L}$ and take a value of about $5 \times 10^{-4}$ a.u. when $r_{s}=2$ (Al valence electron density) and $3 \times 10^{-3}$ a.u. when $r_{s}=1.5$ (Au effective valence electron density). This is basically due to the cancellation of two effects. On the one hand, the number of available vacancies in the final state enhances the total rate when $n_{L}$ is small. On the other hand the rate per spin state increases with $n_{L}$ as the overlap between initial and final state orbitals is larger and the transition energy, which enters in the argument of $S(q, \omega)$, lower. We also find that the rates for $\mathrm{Ne}$ hollow atoms are about six times larger for $\mathrm{Au}\left(r_{s}=1.5\right)$ compared to $\mathrm{Al}\left(r_{s}=2\right)$, 

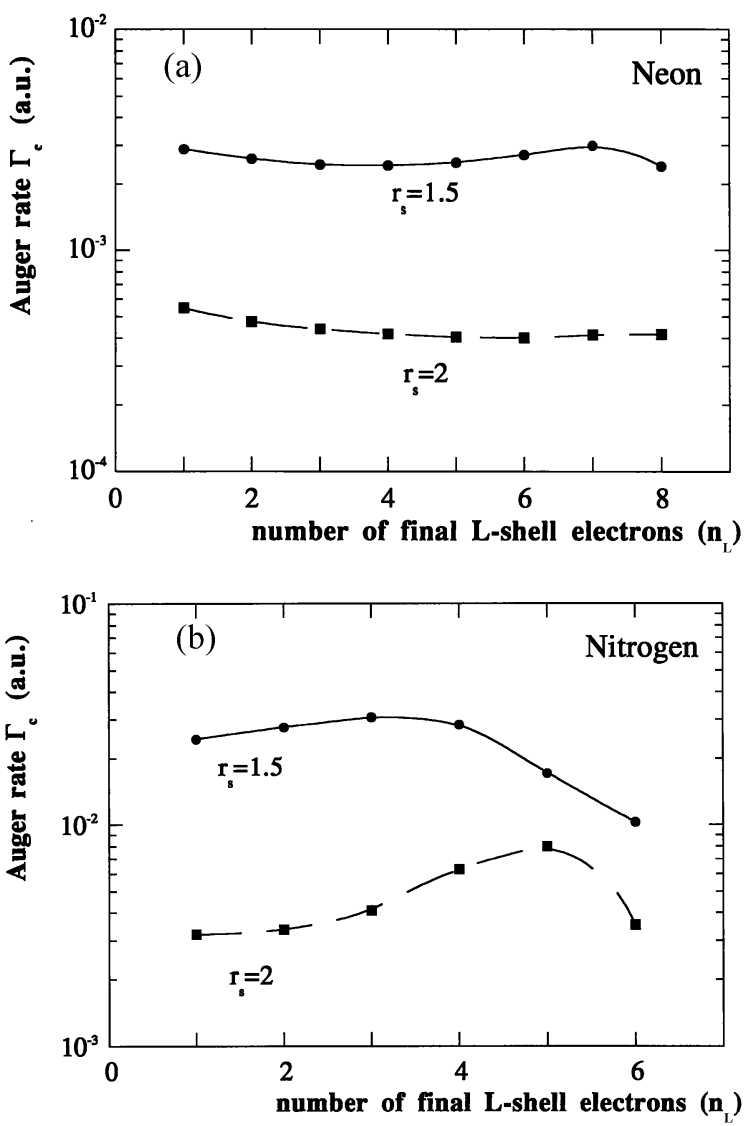

FIG. 2. LCV-Auger rates (in a.u.) as a function of the number of $L$-shell electrons in the final state $\left(n_{L}\right)$ for neon (a) and nitrogen (b) ions in an electron gas. Two different values of $r_{s}$ are considered: $r_{s}=1.5$ (solid line) and $r_{s}=2$ (broken line).

as could be expected from a dependence on the square of the electronic density [27].

In the case of hollow $\mathrm{N}$ atoms the $n_{L}$ dependence of the $L$-shell filling rates is more complex. The binding energy of the $L$-shell levels is strongly dependent on $n_{L}$; in particular the $2 p$ level is very weakly bound when $n_{L}>3$. We find no cancellation between the statistical degeneracy factors and the $n_{L}$ dependence of the rates per spin state in this case. As can be seen in Fig. 3, the transition energy $\Delta E$ that enters in the argument of $S(q, \omega=\Delta E)$ is larger than $\omega_{p}$ for any $n_{L}$ value in the case of $\mathrm{Ne}$, but not in the case of N. For this reason the $S(q, \omega)$ factor determining the rate is monotonically increasing as a function of $n_{L}$ in one case $(\mathrm{Ne})$ and it reaches a maximum in the other $(\mathrm{N})$. This is the main reason why the $n_{L}$ dependence of the rates is so different when comparing $\mathrm{Ne}$ and $\mathrm{N}$ hollow atoms. Comparing the values of the rates that we obtain for $\mathrm{N}$ and $\mathrm{Ne}$ we see that on the average they are about ten times larger for $\mathrm{N}$ than for $\mathrm{Ne}$, as the overlap matrix elements between the continuum states and the bound states is larger the lower the binding energy is.

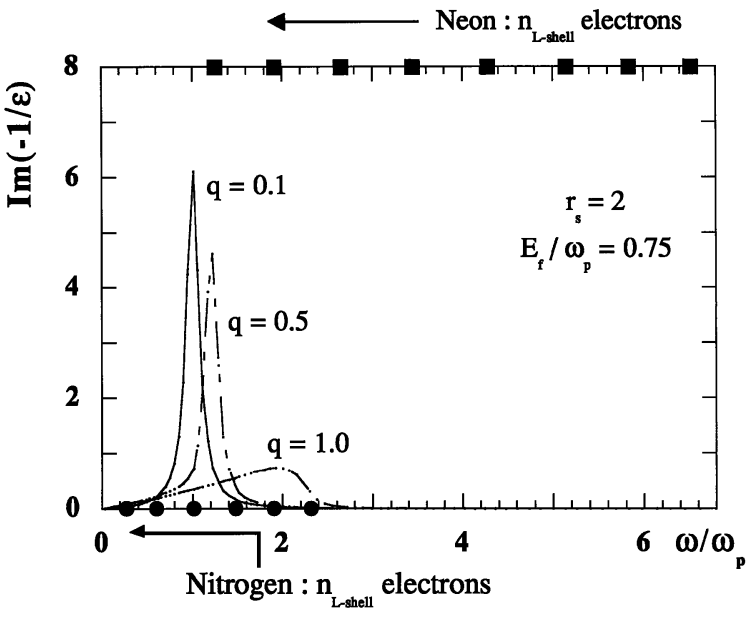

FIG. 3. $\omega$ dependence of the imaginary part of the inverse dielectric function $[\operatorname{Im}(-1 / \varepsilon)]$ for three different $q$ values $\left(0.1,0.5\right.$, and 1.0) in units of the plasma frequency $\omega_{p}$, for $r_{s}=2$. It is related to $S(q, \omega)$ by the relation $\operatorname{Im}(-1 / \varepsilon)=$ $\left(4 \pi^{2} / q^{2}\right) S(q, \omega)$. The dots $(\bullet)$ in the lower $x$ axis represent the $L$-shell binding energy of the $\mathrm{N}$ electrons for $6 \geq n_{L} \geq 1$ [the higher $n_{L}$ the lower the binding]. The squares $(\boldsymbol{\square})$ in the upper $x$ axis represent the $L$-shell binding energy of the $\mathrm{Ne}$ electrons for $8 \geq n_{L} \geq 1$. We see that $\operatorname{Im}(-1 / \varepsilon)$ is essentially a monotonically decreasing function of $\omega$ as $n_{L}$ decreases $(\omega$ increases) in the case of $\mathrm{Ne}$, while it reaches a maximum around $n_{L}=3$ for $\mathrm{N}$. This behavior is reflected in the $n_{L}$ dependence of the rates.

The present results are relevant for the analysis of measured electron spectra and for cascade models [17,28]. For instance, our average value $\left(3 \times 10^{-4}\right.$ a.u. $)$ for the $\mathrm{LCV}$ rate per spin state for the Ne-Al system is close, although somewhat smaller, to the empirical constant value $\left(6.67 \times 10^{-4}\right.$ a.u. $)$ of the rate that was required to explain the Auger electron spectra measured by Stolterfoht et al. [17]. Winter et al. [29] have recently concluded that for the $\mathrm{N}^{6+}$-Au system the large number of low energy electrons that contribute to the electron yield comes from processes that take place at and below the metal surface. This conclusion is based both on a statistical analysis of the electron distributions (indicating that a large number of electrons contribute to the yield with a small escape probability) and on the fact that the electron emission yields are almost independent of projectile velocity. These processes taking place below the surface could well be the LCV-Auger processes for which we find rates of the order of $10^{-2}$ a.u., i.e. large enough to create low energy electrons within the first few target layers.

Another important mechanism for the filling of the $L$ shell is resonant vacancy transfer [16] to the target atom levels. However, even when the resonant vacancy transfer mechanism is more efficient in filling the $L$ shell, Auger transitions may be crucial in opening the resonant vacancy transfer channel by bringing the $L$-shell energy levels into resonance with some target levels. Future experiments may bring information on the relative weight of both 
mechanisms, e.g., with $\mathrm{C}$ targets in which case no target level appears between the $K$ shell and the valence band.

In conclusion, we have determined LCV-Auger rates from first principles by carrying out a nonlinear screening calculation within density functional theory. An average value of $10^{-3}$ a.u. for $\mathrm{Ne}$ and $10^{-2}$ a.u. for $\mathrm{N}$ is found. With our method, calculations for specific projectiletarget combination, as required for the interpretation of experiments, are made possible. Our results are in good agreement with the order of magnitude of the rates used to interpret the most recent electron emission data, and are explained in terms of three governing factors: the background valence electron density ( $r_{s}$ value), the $\omega$ dependence of $S(q, \omega)$, and the one-electron overlap matrix elements appearing in Eq. (1).

The authors gratefully acknowledge help and financial support by Fondo de Cooperacion Euskadi-Aquitania, Ministerio de Educacion y Ciencia, HCM program under Contract No. CHRX-CT93-0103, Euskal Herriko Unibertsitatea, Gipuzkoako Foru Aldundia, and Iberdrola S.A.

*Permanent address: Hahn-Meitner Institut, Glienicker Strasse 100, Berlin, D-14109, Germany.

${ }^{\dagger}$ Also at Unidad Asociada al Instituto de Ciencia de Materiales, C.S.I.C., Cantoblanco, 28049 Madrid, Spain.

‡Permanent address: Laboratoire de Physico-Chimie Théorique, C.N.R.S. et Université de Bordeaux I, 351 Cours de la Libération, 33405 Talence Cedex, France.

[1] M. Grether, A. Spieler, R. Köhrbrück, and N. Stolterfoht, Phys. Rev. A 52, 426 (1995).

[2] S. Hustedt, J. Freese, S. Mähl, W. Heiland, S. Schippers, J. Bleck-Neuhaus, M. Grether, R. Köhrbrück, and N. Stolterfoht, Phys. Rev. A 50, 4993 (1994).

[3] J. Limburg, S. Schippers, R. Hoekstra, R. Morgenstern, H. Kurz, F. Aumayr, and H. P. Winter, Phys. Rev. Lett. 75, 217 (1995).

[4] T. Neidhart, F. Pichler, F. Aumayr, H. P. Winter, M. Schmid, and P. Varga, Phys. Rev. Lett. 74, 5280 (1995).

[5] R. Schuch et al., Phys. Rev. Lett. 70, 1073 (1993).

[6] P. A. Zeijlmans van Emmichoven et al., Phys. Rev. A 47, 3998 (1993).
[7] J. P. Briand et al., Phys. Rev. Lett. 65, 159 (1990).

[8] D. Schneider, M. A. Briere, J. McDonald, and J. Biersack, Radiat. Eff. Defects Solids 127, 113 (1993).

[9] C. Auth, T. Hecht, T. Igel, and H. Winter, Phys. Rev. Lett. 74, 5244 (1995).

[10] F. Aumayr and H. P. Winter, Comments At. Mol. Phys. 29, 275 (1994).

[11] R. Morgenstern and J. Das, Europhys. News 25, 3 (1994).

[12] I. Hughes, Phys. World 8, 43 (1995).

[13] J. Burgdörfer, P. Lerner, and F. W. Meyer, Phys. Rev. A 44, 5674 (1991).

[14] L. Folkerts, S. Schippers, D. M. Zehner, and F. W. Meyer, Phys. Rev. Lett. 74, 2204 (1995).

[15] A. Arnau, P. A. Zeijlmans van Emmichoven, J. I. Juaristi, and E. Zaremba, Nucl. Instrum. Methods Phys. Res., Sect. B 100, 279 (1995).

[16] A. Arnau, R. Köhrbrück, M. Grether, A. Spieler, and N. Stolterfoht, Phys. Rev. A 51, R3399 (1995).

[17] N. Stolterfoht et al., Phys. Rev. A 52, 445 (1995).

[18] W. Heiland, in Interaction of Charged Particles with Solids and Surfaces, edited by A. Gras-Marti et al. (Plenum Press, New York, 1991).

[19] F. Guinea, F. Flores, and P. M. Echenique, Phys. Rev. Lett. 47, 604 (1981); Phys. Rev. B 25, 6209 (1982).

[20] D. Isaacson, in "Compilation of $r_{s}$ values," Radiation and Solid State Laboratory, New York University, 1975.

[21] E. Zaremba, L. M. Sander, H. B. Shore, and J. H. Rose, J. Phys. F 7, 1763 (1977).

[22] P. M. Echenique, R. M. Nieminen, and R. H. Ritchie, Solid State Commun. 37, 779 (1981).

[23] W. Kohn and L. J. Sham, Phys. Rev. 140, A1133 (1965).

[24] P. M. Echenique, F. Flores, and R. H. Ritchie, in Solid State Physics Series, edited by H. Ehrenreich and D. Turnbull (Academic, New York, 1990), Vol. 49, p. 230.

[25] F. Guinea and F. Flores, J. Phys. C 13, 4137 (1980); 14, 2965 (1981).

[26] J. Lindhard, K. Dan, Vidensk. Selsk. Mat. Fys. Medd. 28, 8 (1954).

[27] The dependence of the rate with the square of the background density $\left(\rho_{0}\right)$ comes from the volume of the Fermi sphere $\left(k_{F}^{3}\right)$ times the response function $\left(\omega_{p}^{2}\right)$.

[28] J. Das and R. Morgenstern, Phys. Rev. A 47, R755 (1993).

[29] H. P. Winter, M. Vana, C. Lemell, and F. Aumayr, Nucl. Instrum. Methods Phys. Res., Sect. B (to be published). 